\title{
Financing Strategies to Facilitate Access to High-Cost Anticancer Drugs: A Systematic Review of the Literature
}

\author{
Chanthawat Patikorn $^{1}{ }^{\circledR}$, Suthira Taychakhoonavudh $^{1}$, Rungpetch Sakulbumrungsil $^{1}{ }^{\circledR}$, Dennis Ross-Degnan $^{2}{ }^{\circledR}$, \\ Puree Anantachoti ${ }^{*}{ }^{\star}$
}

\begin{abstract}
Background: Each country manages access to anticancer drugs differently due to variations in the structure and financing of the health system, but a summary of the various strategies used is absent. This study aimed to review and summarize financing strategies implemented across countries to facilitate access to high-cost anticancer drugs.

Methods: We conducted a systematic review of articles referenced in PubMed, Embase, and Web of Science through May 12, 2021. Articles published in the English language from 2000 that describe strategies implemented in different countries to facilitate access to high-cost anticancer drugs were included. Letters, news articles, and proposed strategies were excluded. Quality assessment was not performed as we aimed to summarize the strategies. Data were analyzed by thematic analysis. A review protocol was registered at PROSPERO (CRD42018068616).

Results: The review included 204 studies from 176 countries. Three themes of financing strategies were identified: (1) Basic pharmaceutical reimbursement and pricing policies, (2) Alternative funding strategies specific to high-cost drugs, and (3) Financial assistance for individual patients. Access in most countries depends mainly on basic pharmaceutical reimbursement policies (165 of 176 countries). Apart from that, high-income countries (HICs) tended to use funding strategies targeting high-cost drugs ( $72 \%$ of HICs vs $0 \%-24 \%$ of the rest), such as managed entry agreements (MEAs) or dedicated funds for high-cost drugs. In contrast, lower-income countries tended to implement financial assistance programs for cancer patients as a tool to increase access (32\% of HICs vs $62 \%-79 \%$ of the rest).

Conclusion: Many countries have implemented a combination of strategies to increase access to high-cost anticancer drugs. Most low- and middle-income countries utilized placement of anticancer drugs on a national list of essential medicines and patient assistance programs (PAPs) to facilitate access, while many HICs implemented a broader range of strategies.

Keywords: Expensive, Antineoplastic Drug, Reimbursement

Copyright: @ 2022 The Author(s); Published by Kerman University of Medical Sciences. This is an open-access article distributed under the terms of the Creative Commons Attribution License (https://creativecommons.org/licenses/ by/4.0), which permits unrestricted use, distribution, and reproduction in any medium, provided the original work is properly cited.

Citation: Patikorn C, Taychakhoonavudh S, Sakulbumrungsil R, Ross-Degnan D, Anantachoti P. Financing strategies to facilitate access to high-cost anticancer drugs: a systematic review of the literature. Int $J$ Health Policy Manag. 2022;11(9):1625-1634. doi:10.34172/ijhpm.2021.138
\end{abstract}

Article History:

Received: 5 October 2021

Accepted: 21 September 2021 ePublished: 22 September 2021

\footnotetext{
*Correspondence to:

Puree Anantachoti

Email: puree.a@chula.ac.th
}

\section{Background}

Cancer is a leading cause of death with 9.6 million deaths worldwide in 2018. ${ }^{1}$ Many effective therapies are available for cancer including surgery, radiation, and anticancer drugs. However, healthcare systems face challenges in providing access to anticancer drugs for patients who need them while controlling the overall cost of cancer care which has been increasing rapidly over the past two decades. Global expenditures on cancer therapies and supportive care drugs reached US $\$ 113$ billion in 2016 and were expected to increase to more than US $\$ 137$ billion by $2021 .^{2}$

Anticancer drugs are highly priced to reflect cost of lengthy research and development of both successful and unsuccessful drugs. Moreover, the use of anticancer drugs aimed for rare cancers are limited to relatively small number of patients. Because the life-threatening nature of cancer, patients are likely to express more willingness to pay for anticancer drugs, even though the benefits of prolongation of survival are limited. ${ }^{3}$ Therefore, the pharmaceutical companies could set price of anticancer drugs as high as the market could bear maximize profitable returns. It was found that the median annual price of anticancer drugs has been increasing from US\$12000 to more than US\$120000 over the past two decades. ${ }^{2}$ The recently developed anticancer drugs such as CAR T-cell therapy are even more expensive with treatment cost up to US\$500000 per year. ${ }^{4}$ Given the rising costs of anticancer drugs, payers are unlikely to provide unconditional reimbursement as a long-term solution. To stay within a pharmaceutical budget, reimbursements are often restricted to indications that provide demonstrated value for money. However, such restrictions can deny access to potentially useful therapy for patients in high need.

Limited access to high-cost anticancer drugs is not only an issue in low- and middle-income countries ${ }^{2,5,6}$ but in high- 
income countries (HICs) as well. ${ }^{7,8}$ It was estimated in 2011 that only $15 \%$ of patients who lived in low- and middleincome countries in the Association of Southeast Asian Nations (ASEAN) had access to anticancer drugs compared with $55 \%$ of patients in Singapore, a HIC in ASEAN. ${ }^{5}$ Each country manages access to anticancer drugs differently due to variations in the structure and financing of the health system.

Previous studies have mostly summarized strategies to facilitate access to high-cost anticancer drugs in a single country ${ }^{7,9}$ or a group of countries, ${ }^{2,5,8,10}$ but a summary of this disassembled evidence is absent. For a country to establish informed and effective strategies to deal with access to anticancer drugs, it is important to know the overall landscape of strategies implemented across the world, including not only HICs, but low- and middle-income countries as well. Therefore, the objective of this study was to review and summarize strategies implemented across countries at different income levels to facilitate access to high-cost anticancer drugs.

\section{Methods \\ Search Strategy and Selection Criteria}

This systematic review was conducted to summarize the financing strategies implemented in any countries to facilitate patients with cancer to access to anticancer drugs. This systematic review reported following the Preferred Reporting Items for Systematic reviews and Meta-Analyses (PRISMA) guideline $^{11}$ (Supplementary file 1). A protocol of this review was registered at PROSPERO - CRD42018068616.

The literature search was undertaken in May 12, 2021, using PubMed, Embase, and Web of Science. The search strategy used combinations of terms including "Policy," "Program," "Access," "Cancer" and "Drugs" (Supplementary file 2). A search of the grey literature was not feasible to perform due to the lack of resources for non-English language.

For purposes of this review, strategy was defined as any policies, policy instruments, programs, or activities established with the aim to facilitate access to anticancer drugs. We included a strategy when the focus of the article was anticancer drugs or if the authors stated that a general strategy had been implemented to facilitate access to anticancer drugs.

The eligible studies are full-text articles published in the English language from 2000 that describe financing strategies implemented in different countries to facilitate access to highcost anticancer drugs. Letters, news articles, and proposed strategies were excluded.

After duplicates were removed, two reviewers (CP and ST) independently screened abstracts and titles for relevance. The full-text articles were independently selected by two reviewers (CP and ST).

\section{Data Extraction}

One reviewer (CP) extracted data from selected studies in a data extraction form. The following data were extracted from the included studies: country, name of strategy, objective of strategy, description of strategy, initial year of implementation, initiators, and responsible organizations. The selected studies were then divided among the second (ST) and a third reviewer
(PA) for cross-check of extracted data. Any discrepancies were resolved through a consensus discussion among reviewers.

\section{Quality Assessment}

Quality and risk of bias assessment was not performed since this study aimed to describe and summarize the financing strategies.

\section{Data Analysis}

The extracted data were analyzed using thematic analysis. One reviewer (CP) initially constructed coding framework by categorizing the extracted financing strategies based on how they aimed to facilitate access to anticancer drugs by targeting insurance coverage status, reimbursement, or price. Themes were developed from the coding process of the extracted data by adding new types of strategies and relevant sub-types, and refining the synthesized themes until saturation was reached when no more themes were identified. Subsequently, the synthesized themes were then discussed among researchers (RS, DRD, ST, and PA) until the themes were finalized.

The identified countries were classified by the World Bank's income levels to analyze the variation in the implementation of financing strategies across income levels. ${ }^{12}$ Results were summarized and presented by theme.

Results

The search identified 3096 candidate studies of which 204 were included (Figure 1). A total of 176 countries was identified, comprising 53 HICs (30\%), 50 upper middleincome countries (U-MICs) (28\%), 47 lower middle-income countries (L-MICs) (27\%), and 26 low-income countries (LICs) (15\%). There were 172 studies describing strategies implemented in HICs, 56 studies in U-MICs, 25 studies in L-MICs, and 11 studies in LICs.

We identified the 3 major themes with 12 sub-themes concerning strategies to facilitate patient access to highcost anticancer drugs (Figure 2). Strategies in each country were summarized - (Supplementary file 3). The absence of strategies in countries indicated that they were not mentioned in the literature. Strategies were organized in the following categories:

1) Basic pharmaceutical reimbursement and pricing policies included features of the basic reimbursement and pricing system in countries tailored to provide access to anticancer drugs to their population;

2) Funding strategies specific to high-cost drugs included add-on strategies used specifically to provide access to high-cost drugs such as orphan or anticancer drugs when the basic pharmaceutical reimbursement and pricing policies are insufficient;

3) Financial assistance for individual patients included add-on strategies intended to provide financial support for cancer patients by either governmental or non-governmental organizations.

Among these, basic pharmaceutical reimbursement and pricing policies were the most commonly utilized policy ( $94 \%$ of 176 countries). Apart from the basic pharmaceutical reimbursement and pricing policies, HICs tended to use 


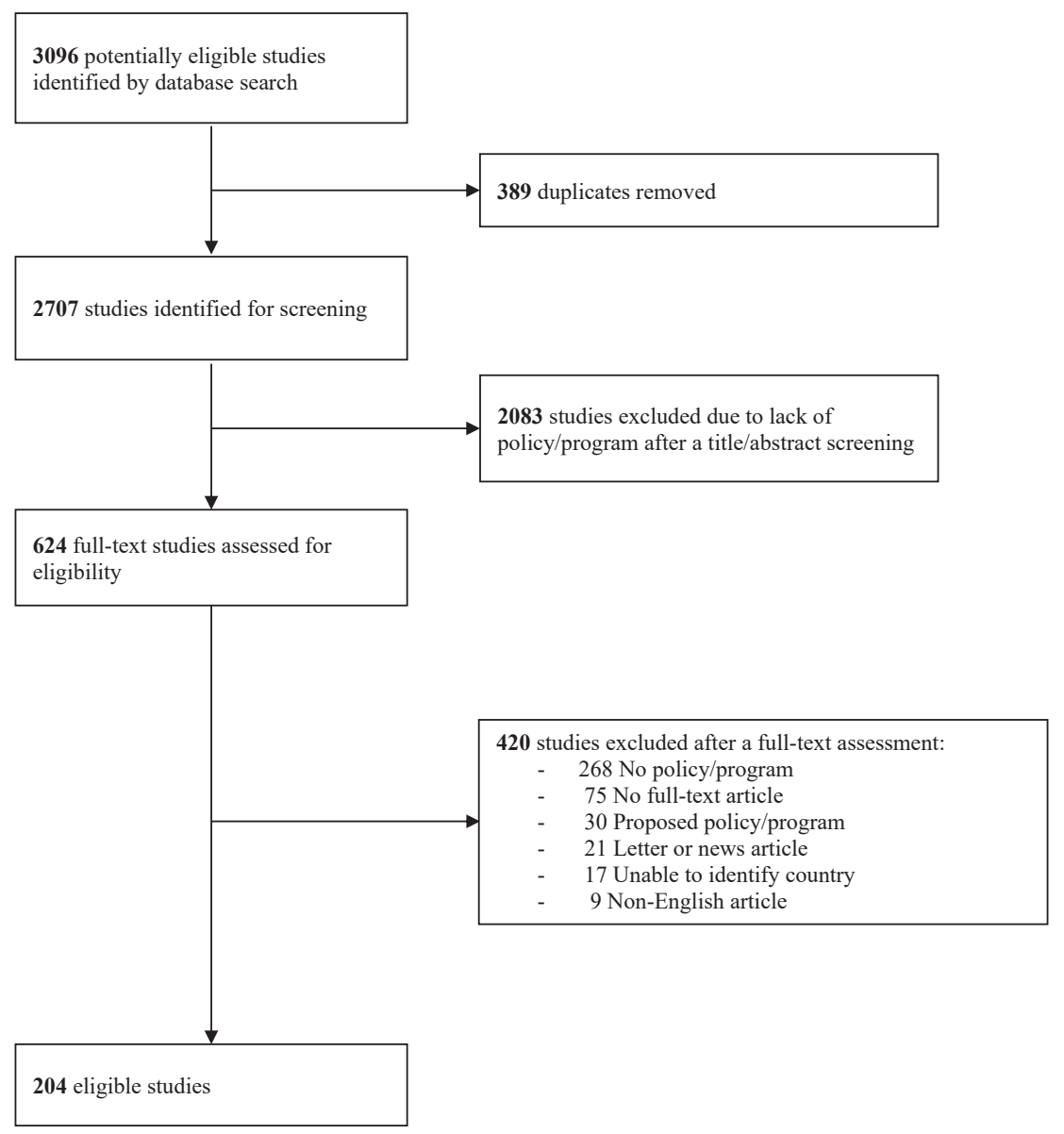

Figure 1. Study Selection Flow. Note: To ease presentation of the study selection process, the reasons for excluding studies after a full-text assessment were only described with the main reason.

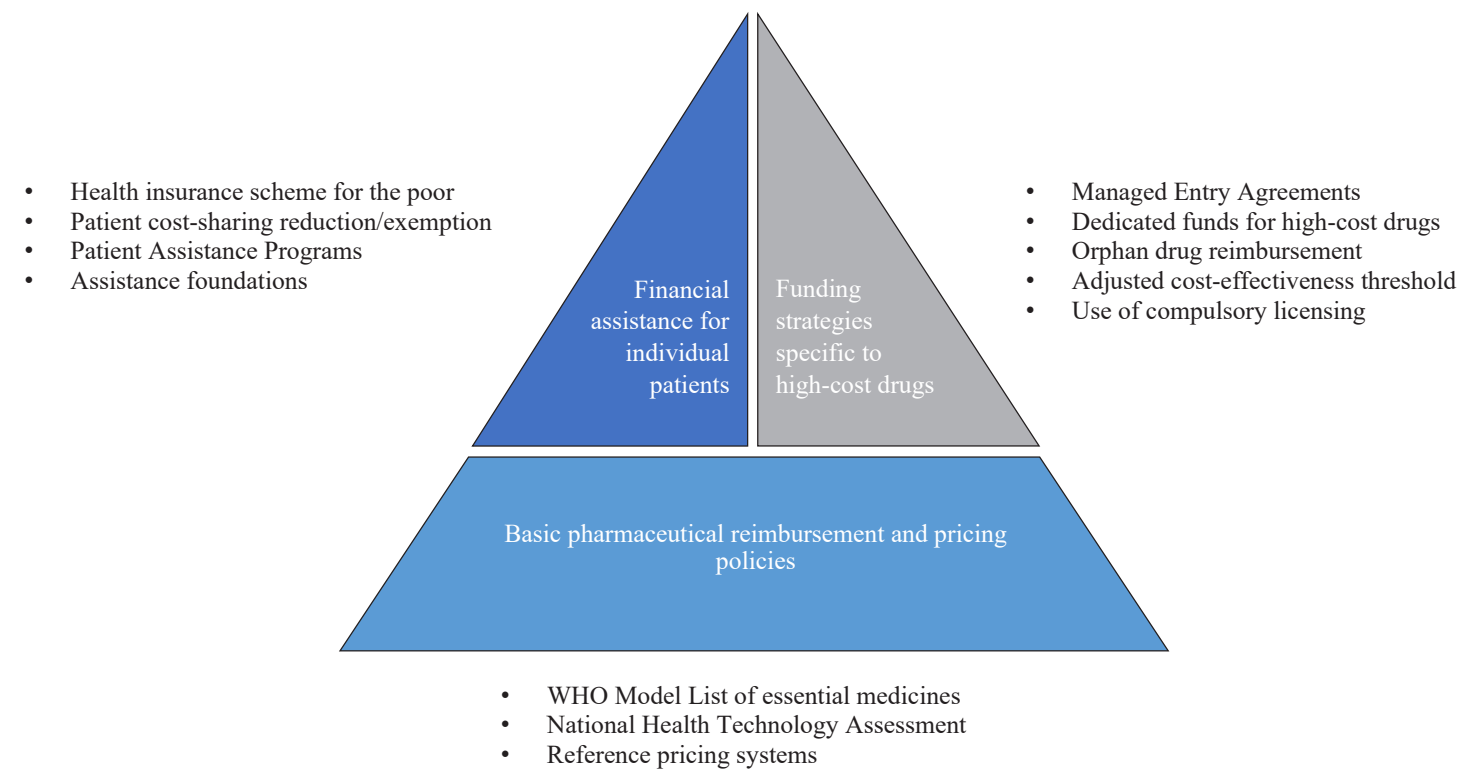

Figure 2. Themes and Sub-themes of Financing Strategies to Facilitate Access to High-Cost Anticancer Drugs. Basic pharmaceutical reimbursement and pricing policies included features of the basic reimbursement and pricing system in countries tailored to provide access to anticancer drugs to their population; Funding strategies specific to high-cost drugs included add-on strategies used specifically to provide access to high-cost drugs such as orphan or anticancer drugs when the basic pharmaceutical reimbursement and pricing policies were insufficient; Financial assistance for individual patients included add-on strategies intended to provide financial support for individual patients by either governmental or non-governmental organizations. Abbreviation: WHO, World Health Organization. 
funding strategies for high-cost drugs (72\% of HICs vs $0 \%-24 \%$ of the rest) while the rest implemented financial assistance programs for cancer patients as a tool to increase access (32\% of HICs vs $62 \%-79 \%$ of the rest) as shown in Table 1 and Table 2.

Basic Pharmaceutical Reimbursement and Pricing Policies Reimbursement and pricing policies are used by many countries to increase access to expensive medicines that are beyond most patients' ability to pay. ${ }^{13,14}$ Reimbursement decisions are often made at the national level especially in countries where public health insurance is available. A national list of reimbursable medicines is also often referred to as a national drug formulary or a national list of essential medicines. ${ }^{9,15,16}$ In some countries, sub-national reimbursement schemes exist in which local, provincial or regional governments have reimbursement lists that are independent (eg, Canada) ${ }^{17}$ or that supplement the national reimbursement list (eg, China). ${ }^{18}$

When local governments independently determine their own benefit scheme, significant geographic variations in access to anticancer drugs can occur, leading to a "postcode prescribing" phenomenon in which a patient's access depends on where they live. For example, in Canada, only 7 out of 115 anticancer drugs were found to be available in all ten provinces. ${ }^{19}$ In Italy, although pricing and reimbursement decisions are made nationally, regional governments can charge different co-payments leading to price variations across the country. ${ }^{10}$

Decisions to include anticancer drugs on national formularies are made with the guidance of several policy tools including the World Health Organization Model Lists of Essential Medicines (WHO-EML), health technology assessments (HTAs), and various price determination tools.

\section{WHO Model Lists of Essential Medicines}

Since 1977, WHO has published the WHO-EML to guide countries in prioritizing access to essential medicines. In 2017, the WHO-EML included 40 anticancer drugs. ${ }^{20}$ WHO-EMLs are the most widely-used tools to increase access to anticancer drugs ( $77 \%$ of countries); however, their use is less common in HICs (45\% of countries). U-MICs, L-MICs, and LICs (90\%, $91 \%$, and $92 \%$ of countries, respectively) used the WHO-EML as a guide to select medicines to include in their national drug formularies especially countries with low capacities and capabilities. However, the WHO-EML covers limited number of types of cancer with the median concordance between their national formularies in these countries and the WHO-EML was $42 \%{ }^{15}$

\section{National Health Technology Assessment}

HTA is a multidisciplinary process for summarizing and evaluating information regarding efficacy, safety, costeffectiveness (CE), and ethical and societal preferences for medical therapies and technologies to inform reimbursement decisions by payers. HTA was reported as a tool for reimbursement decisions in over half of HICs (75\% of countries), with much lower use in U-MICs, L-MICs, and LICs $(40 \%, 9 \%$, and $0 \%$, respectively).

Each country has its own national HTA organization, process, and decision criteria. ${ }^{21} \mathrm{CE}$ analyses which show value for money in the context of an individual health system are part of the HTA in some countries to efficiently select drugs for reimbursement and ensure affordability and sustainability of the health system. Countries may explicitly define a target CE threshold. 2,22 Some countries, especially LICs and MICs, may comply with the WHO recommendation of three times the gross domestic product per capita as the CE threshold. ${ }^{2}$

Since the HTA reviewing process can be lengthy, Canada and Denmark created special HTA pathway for anticancer drugs. In Canada, the pan-Canadian Oncology Drug Review did not publicly define an explicit CE threshold, which could result in decisions to reimburse high-cost anticancer drugs with relatively poor CE. ${ }^{17}$ In Denmark, the National Board of Health was once established in 2008 to separately review anticancer drugs for national reimbursement. The Danish Centre for Health Technology Assessment could rapidly review anticancer drugs within three months, compared with the normal process of one to two years for non-anticancer drugs. However, Danish Centre for Health Technology Assessment ceased to exist in 2012 when HTA in Denmark was shifted from the central to the regional level. ${ }^{21}$ These special HTA pathways facilitate patient access to high-cost anticancer drugs through prioritization of oncology before other therapeutic areas.

HTA results, especially CE analyses and budget impact analyses, facilitate price negotiations and reimbursement price setting of a new product according to its added therapeutic value compared to existing treatments. ${ }^{9,23}$ Comparison criteria include, but are not limited to, quality-adjusted life years (QALYs) gained, degree of innovation, level of unmet need, lack of an effective alternative treatment, and burden of

Table 1. Themes of Financing Strategies by Country Income Level

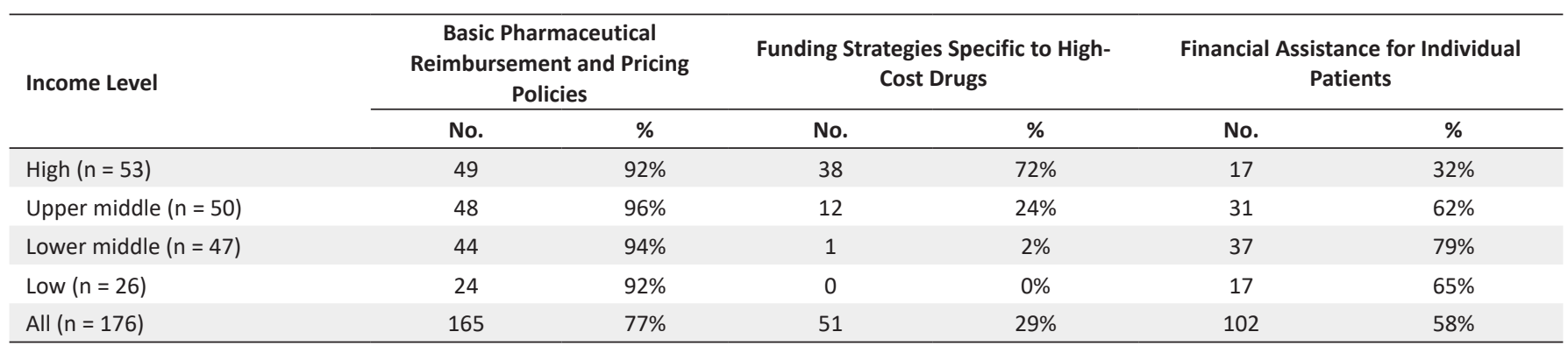


Patikorn et al

Table 2. Sub-themes of Financing Strategies by Country Income Level

\begin{tabular}{|c|c|c|c|c|c|c|c|c|c|c|c|c|c|c|c|c|c|c|c|c|c|c|c|c|}
\hline \multirow[t]{3}{*}{ Country } & \multicolumn{6}{|c|}{$\begin{array}{l}\text { Basic Pharmaceutical Reimbursement and } \\
\text { Pricing Policies }\end{array}$} & \multicolumn{10}{|c|}{ Funding Strategies Specific to High-Cost Drugs } & \multicolumn{8}{|c|}{ Financial assistance for individual patients } \\
\hline & \multicolumn{2}{|c|}{ WHO-EML } & \multicolumn{2}{|c|}{$\begin{array}{l}\text { National Health } \\
\text { Technology } \\
\text { Assessment }\end{array}$} & \multicolumn{2}{|c|}{$\begin{array}{l}\text { Reference } \\
\text { Pricing } \\
\text { Systems }\end{array}$} & \multicolumn{2}{|c|}{ MEA } & \multicolumn{2}{|c|}{$\begin{array}{l}\text { Dedicated } \\
\text { Funds for High- } \\
\text { Cost Drugs }\end{array}$} & \multicolumn{2}{|c|}{$\begin{array}{l}\text { Orphan Drug } \\
\text { Reimbursement }\end{array}$} & \multicolumn{2}{|c|}{$\begin{array}{l}\text { Adjusted Cost- } \\
\text { Effectiveness } \\
\text { Threshold }\end{array}$} & \multicolumn{2}{|c|}{$\begin{array}{l}\text { Use of } \\
\text { Compulsory } \\
\text { Licensing }\end{array}$} & \multicolumn{2}{|c|}{$\begin{array}{l}\text { Health } \\
\text { Insurance } \\
\text { Scheme for the } \\
\text { Poor }\end{array}$} & \multicolumn{2}{|c|}{$\begin{array}{l}\text { Patient Cost-Sharing } \\
\text { Reduction/Exemption }\end{array}$} & \multicolumn{2}{|l|}{ PAP } & \multicolumn{2}{|c|}{$\begin{array}{l}\text { Assistance } \\
\text { Foundations }\end{array}$} \\
\hline & No. & $\%$ & No. & $\%$ & No. & $\%$ & No. & $\%$ & No. & $\%$ & No. & $\%$ & No. & $\%$ & No. & $\%$ & No. & $\%$ & No. & $\%$ & No. & $\%$ & No. & $\%$ \\
\hline High $(n=53)$ & 24 & 45 & 40 & 75 & 26 & 49 & 31 & 58 & 18 & 34 & 16 & 30 & 6 & 11 & 1 & 2 & 1 & 2 & 4 & 8 & 13 & 25 & 2 & 4 \\
\hline Upper middle $(n=50)$ & 45 & 90 & 20 & 40 & 5 & 10 & 7 & 14 & 7 & 14 & 3 & 6 & 0 & 0 & 1 & 2 & 3 & 6 & 2 & 4 & 30 & 60 & 0 & 0 \\
\hline Lower middle $(n=47)$ & 43 & 91 & 4 & 9 & 2 & 4 & 0 & 0 & 0 & 0 & 0 & 0 & 0 & 0 & 1 & 2 & 1 & 2 & 0 & 0 & 37 & 79 & 0 & 0 \\
\hline Low $(n=26)$ & 24 & 92 & 0 & 0 & 0 & 0 & 0 & 0 & 0 & 0 & 0 & 0 & 0 & 0 & 0 & 0 & 0 & 0 & 0 & 0 & 17 & 65 & 0 & 0 \\
\hline All $(n=176)$ & 136 & 77 & 64 & 36 & 33 & 19 & 38 & 22 & 25 & 14 & 19 & 11 & 6 & 3 & 3 & 2 & 5 & 3 & 6 & 3 & 97 & 55 & 2 & 1 \\
\hline
\end{tabular}

Abbreviations: PAPs, patient assistance programs; MEA, managed entry agreement; WHO-EML, World Health Organization Model Lists of Essential Medicines. 
disease targeted by the product. This strategy is called "Valuebased pricing" in Sweden and the United Kingdom. ${ }^{24,25}$ Prices of anticancer drugs may be set in reference to the country's CE threshold. A system using an explicit or implicit CE threshold seeks to limit spending on drugs with low therapeutic value, and also incentivizes development of drugs with more added value. For example, in South Korea, there is a two-stage process for price negotiations, first an HTA-specific price negotiation process to lower price to a level consistent with the $\mathrm{CE}$ analysis followed by an obligatory price negotiation process with the South Korean payer. ${ }^{23}$ Although price negotiations could lower prices substantially, details in price negotiations in most countries were seldom disclosed.

\section{Reference Pricing Systems}

The use of reference pricing was reported by HICs (49\% of countries) with much lower use in U-MICs, L-MICs, and LICs $(10 \%, 4 \%$, and $0 \%$, respectively). External reference pricing sets product prices based on a formula (eg, average, median, or other summary) using prices from several reference countries, while internal reference pricing sets a single reimbursement price for a group of drugs clustered by the mechanism of action, molecular similarity, or sometimes therapeutic effect. Some countries utilize external reference pricing to set the price of high-cost anticancer drugs, especially European countries where maximum prices were set based on the average price in other European countries which led to price transparency and information sharing across countries. ${ }^{10,24,26}$ Multinational pharmaceutical companies try to circumvent external reference pricing by limiting public information about their real prices (eg, by using confidential managed entry agreements, $\mathrm{MEAs}^{27}$ ) or delaying or avoiding product launches in countries with rigorous external reference pricing. ${ }^{23}$

Internal reference pricing systems are also utilized in many European countries. ${ }^{10,28}$ For example, in Germany and the Netherlands, anticancer drugs with limited added benefits compared to existing therapies are priced at the level of similar drugs in a therapeutic class. ${ }^{28,29}$ This system allows greater patient access to several anticancer drugs within the same therapeutic class.

\section{Funding Strategies Specific to High-Cost Drugs}

Included in this theme are specific strategies used to provide access to high-cost anticancer drugs and other high-cost treatments when the basic reimbursement and pricing policies proved to be insufficient. Most strategies are implemented by major public insurance payers using different tools and criteria. These funding strategies are not consistent across countries; some countries may use one strategy in conjunction with specific criteria while other countries may use the same strategy with different criteria.

\section{Managed Entry Agreements}

MEAs are one of the most common funding strategies found in the literature. From 176 countries reviewed, 38 (22\%) reported the use of MEAs to increase access to highcost anticancer drugs. MEAs are a policy tool utilized when reimbursement decisions cannot be made due to uncertainties about clinical evidence, financial impacts or CE. ${ }^{27}$ MEAs are known as patient access schemes in the United Kingdom, ${ }^{30}$ managed access programs in Australia, ${ }^{31}$ and coverage with evidence development in the Netherlands. ${ }^{29}$ There are two main types of MEAs: performance-based MEAs that link drug reimbursement to a drug's performance or patient outcomes and financial-based MEAs that indirectly lower drug prices through simple discounts, price-volume agreements, or rebates. Some MEAs utilize both. ${ }^{27,30}$

MEAs facilitate access to high-cost anticancer drugs which would otherwise not be reimbursed at their offered prices by health system payers. The types and designs of MEAs vary from country to country. For example, most MEAs in Italy are performance-based with a refund for non-responders at the individual patient level while the majority of MEAs in the United Kingdom are financial-based with simple discounts. ${ }^{27}$

MEAs have been increasingly used over time in HICs. One of the attractive attributes of MEAs is that significant price reductions can be achieved by payers while pharmaceutical companies can keep the negotiated net price undeclared. This hinders the effectiveness of external reference pricing since listed prices are not the real prices used. ${ }^{27}$

\section{Dedicated Funds for High-Cost Drugs}

Some countries that have a societal preference for treatment of cancer over other diseases establish dedicated funds to subsidize access to high-cost anticancer drugs. Dedicated funds include special national budgets for high-cost drugs, additional payments for high-cost drugs, or special programs to provide access to drugs awaiting reimbursement decision. ${ }^{9,10,25,32}$ Dedicated funds for high-cost anticancer drugs were reported in 25 (14\%) of countries, of which 18 are HICs.

The well-known Cancer Drugs Fund (CDF) has been established in England in 2011. Drugs subsidized under the $\mathrm{CDF}$ are those receiving a negative recommendation from The National Institute for Health and Care Excellence (NICE) or those still in the reimbursement approval process. ${ }^{25}$ Due to rapidly increasing budgets, the CDF has been integrated into the NICE appraisal program since 2016. Drugs under the CDF will receive coverage with evidence development for two years with the chances of being delisted if further evidence shows no additional benefits or unresolved uncertainties. ${ }^{33}$ Therefore, dedicated funds require strong health system, technical capacity, and fund to appropriately decide which drugs to support and design monitoring system to generate evidence for further revision of the funds.

\section{Orphan Drug Reimbursement}

Anticancer drugs that affect only a small proportion of the population or rare diseases are sometimes classified as orphan drugs, although the criteria vary from country to country. We found 16 HICs and 3 U-MICs reporting the use of orphan drug reimbursement. Some countries adjust CE thresholds to facilitate a reimbursement decision or provide full coverage without co-payments for orphan drugs. ${ }^{32,34,35}$

Use of orphan drug status not only facilitates better access to 
medicine for patients with rare diseases but also incentivizes pharmaceutical companies to research and develop these drugs. Even though these criteria have not been specifically established for cancers, anticancer drugs would often be eligible.

\section{Adjusted Cost-Effectiveness Threshold}

Because most innovative anticancer drugs are high-cost, they exceed most countries' CE thresholds which would normally result in denial of reimbursement. However, six HICs (Australia, South Korea, the Netherlands, Slovakia, Sweden, and the United Kingdom) report use of higher CE thresholds as an add-on policy to the normal HTA process under certain circumstances, including expected number of patients, disease severity, medical need, lack of effective treatment, societal values, and expected impacts..$^{10,21,23,35,36}$ These adjustments of CE thresholds are more common for anticancer drugs.

In Australia, the "Rule of Rescue" allows reimbursement of cost-ineffective drugs treating severe and progressive diseases that affect a small number of patients when there is no existing alternative treatment. ${ }^{21}$ In the United Kingdom, the "End-oflife Criteria" were created specifically to fund life-prolonging drugs to treat diseases with short life expectancy by allowing incremental $\mathrm{CE}$ ratio beyond the usual $\mathrm{CE}$ threshold of $£ 30000$. These criteria have led to a higher proportion of anticancer drugs approved for reimbursement. ${ }^{35}$

Many countries allow higher CE thresholds depending on disease severity. However, the extent of CE threshold adjustments in most countries was not explicitly stated, with the exception of the Netherlands where the CE threshold can be adjusted between $€ 20000$ and $€ 80000 / Q A L Y$ according to disease severity and medical need. ${ }^{32}$ Drugs reimbursed under the adjusted CE threshold are listed as innovative drugs and hospitals receive add-on payments for these high-cost drugs. ${ }^{32}$

\section{Use of Compulsory Licensing}

The World Trade Organization Trade-Related Aspects of Intellectual Rights agreement allows any country with public urgent need to issue a compulsory license without consent from the patent holder to produce a generic drug. Also, the Doha Declaration allows countries without competency to produce local generic drug to import from other countries. Before issuing a compulsory license, the government may request a voluntary license from the pharmaceutical manufacturer. ${ }^{5}$ Compulsory licenses for anticancer drugs were reported only in Italy, India, and Thailand. ${ }^{5,9,37}$ In Thailand, the government issued compulsory licenses for three anticancer drugs (docetaxel, letrozole, and erlotinib) in 2008 to purchase generic drugs from India which resulted in a cost saving of more than US\$140 million over 5 years. ${ }^{9}$ Compulsory licensing can significantly increase patient access to high-cost drugs, or serve as a negotiating tool to lower manufacturers' drug prices, as seen in Colombia. ${ }^{2}$

\section{Financial Assistance for Cancer Patients}

Some countries have developed strategies to help patients pay for their anticancer drugs out of pocket, either because they are not included in insurance reimbursement list or the patients are not enrolled in any health insurance scheme.

\section{Health Insurance Scheme for the Poor}

We found five countries (the United States, China, Mexico, Russia, and India) where governments have established separate health insurance schemes to provide access to anticancer drugs for the poor or uninsured. Each scheme varied in its eligibility criteria, drug formulary, and benefits package. However, the schemes still failed to provide access to essential anticancer drugs and had insufficient coverage of the total costs of therapy. ${ }^{18,38,39}$

\section{Patient Cost-Sharing Reduction/Exemption}

When high-cost anticancer drugs are not fully covered, patients face high cost-sharing. Treatments can be provided to eligible patients with co-payment reductions or exemptions. Such reductions/exemptions from cost-sharing were found in six countries including Croatia, France, the Netherlands, the United States, China, and Iran. However, most of these reductions/exemptions are not specific for anticancer drugs. ${ }^{10,18}$

\section{Patient Assistance Programs}

Some pharmaceutical companies have developed patient assistance programs (PAPs) which donate anticancer drugs to patients who cannot afford them for low or no cost. PAPs are one of the most common ways that patients can access highcost anticancer medicines when regular access through health insurance is limited. The programs and their regulation vary from country to country. PAPs were found in 97 countries (55\% of 176 countries), comprising mainly low- and middleincome countries (84 of 176 countries).

The most successful PAP is the Glivec/Gleevec International Patient Assistance Program (GIPAP) supported by Novartis. Since 2001, GIPAP had provided access to 63000 patients in 93 countries who were otherwise unable to access imatinib. ${ }^{40,41}$

Pharmaceutical companies use PAPs as one way to mask the real net price of their products in a country. They are also criticized as special marketing arrangements that offer indirect discounts, for example, the "buy 3 get 1 free" program reported by Sruamsiri et al in Thailand. ${ }^{9}$

\section{Assistance Foundations}

As a last resort, patients may sometimes access affordable high-cost anticancer drugs through foundations or charities. The foundations were reported in Hong Kong and the United States. In Hong Kong, a charity called the Hong Kong AntiCancer Society has been established to assist patients who need financial support by giving cash subsidies and obtaining free drugs from pharmaceutical companies. ${ }^{42}$ In the United States, patient foundations, such as the Patient Access Network Foundation, provide financial support to Medicare patients. However, not every patient can obtain financial support, as each foundation has its own eligibility criteria, eligible drugs and diseases, and limited budget. ${ }^{43,44}$

\section{Discussion}

This systematic review is the first study to date which 
comprehensively summarizes financing strategies in 176 countries implemented to facilitate access to high-cost anticancer drugs during the last two decades. Although this review was focused on anticancer drugs, the financing strategies described in this study are frequently implemented for other high-cost drugs as well.

For countries that face difficulty in ensuring access to high-cost anticancer drugs, this review can provide decisionmakers with a broad view of the overall landscape of strategies to facilitate access to anticancer drugs in countries at different economic levels. However, which strategy will best serve a specific population will depend on the context of the health system in each country because there is no single strategy that could effectively facilitate access to anticancer drugs in every country. Likewise, different approaches are needed to improve access to anticancer drugs complementarily.

Our systematic review found that a range of interacting financing strategies have been implemented to facilitate access to anticancer drugs, as shown in Figure 2. Basic pharmaceutical reimbursement and pricing policies serve as the fundamental first tier of strategies to provide access to high-cost anticancer drugs. HTA assists reimbursement decision making by evaluating the evidence and value for money of drugs under the country's CE criteria. Countries use different pricing approaches to negotiate with manufacturers in order to encourage them to lower drug costs to an acceptable level. Most approaches are linked to listing specific drugs on the country's reimbursement list, with many lower income countries using the WHO-EML as a reference.

When basic pharmaceutical and pricing policies are not feasible or when they result in insufficient access to highcost anticancer drugs, funding strategies specific to high-cost drugs and financial assistance for cancer patients may help to fill the gap in access. MEAs are beneficial primarily at an early stage of adding high-cost anticancer drugs to an insurance reimbursement list. In some circumstances, compulsory licenses can provide early access to generic anticancer drugs, which can result in substantial cost savings. Some PAPs provide financial assistance for patients who would otherwise not be able to afford needed anticancer drugs or when other strategies were not available to provide access. However, these alternative access strategies require some features of the basic pharmaceutical and pricing policies especially HTA which could be considered as the foundation to thoroughly and effectively develop informed strategies. For example, MEAs could not be efficiently implemented with appropriate designs if there is no HTA process in place to depict the uncertainties surrounding the decision to reimburse health technologies with could be worthily extended beyond costs per QALYs to include other values of anticancer drugs such as productivity, family spillovers, value of hope, and equity. ${ }^{45}$ Similarly, dedicated funds for high-cost drugs, orphan drug reimbursement, adjusted CE threshold, and use of compulsory licensing also require the existing HTA process.

Our results indicate that each country implements various policies and programs according to unique health system objectives, in light of concerns about health system and patient affordability. HICs mainly implemented both basic pharmaceutical reimbursement and pricing policies and targeted funding strategies specific to anticancer drugs. In contrast, lower income economies mostly relied on financial assistance for cancer patients to supplement basic pharmaceutical reimbursement structures.

It is unarguably that cancer affects patient's and their family life tremendously both in terms of life lost and quality of life. Increasing access to anticancer that could save lives, thus, became an issue that many health policy makers are trying to achieve. It should be noted here that providing access to highcost anticancer drugs will increase burden on the already constrained healthcare budget. This will raise concerns on equity, affordability, and sustainability of such access especially the opportunity costs of providing access to cancer instead of other diseases.

Healthcare financing is one of the several core functions of the healthcare system. However, each healthcare system places different values on the treatment outcome of the patient. Even most financing strategies summarized in this study have an aim to achieve access to anticancer, the values of the treatment these financing strategies have tried to evaluate may be different. Therefore, to effectively adopt financing strategies from other countries to facilitate access to high-cost anticancer drugs, policy makers should thoroughly consider the potential benefits and challenges of financing strategy choices along with their country's surrounding environment such as health insurance system, economy, infrastructure of health system, information system, capacity of human resources, and the existing policies. For instance, the priority of a country without national public insurance system might be to develop a national insurance for their population followed by supporting systems such as HTA before proceeding with alternative access pathways. Moreover, strong information system is necessary to support informed decisions to facilitate access to anticancer drugs not only selection process but also drug use monitoring to further refine the local treatment protocol in each country.

Some limitations of this review and recommendation for future research are worth mentioning. One major limitation of this review is publication bias since we included only studies published in peer-reviewed journals in the English language and a search of the grey literature was not performed. Authors from HICs are much more likely to write on this topic for English-language journals. Limiting the review to published literature ensured a higher quality of the included studies, but constrained its ability to fully capture strategies that were not reported in the academic literature or evolving implementation details of the identified strategies. Some strategies might have been used only once or now ceased to exist. Some strategies might also have been replaced by the other strategies but were not explicitly reported in the published literature. For example, periodic price review of highly priced anticancer drugs may have been used in several countries to adjust the prices based on their actual budget impact but were not explicitly mentioned in the included articles. Moreover, the in-depth synthesis the social and economic environment surrounding the strategies in each country was not feasible given the data reported in the 
published literature. Therefore, a survey with key informants in each country would likely capture a more complete set of strategies to facilitate access to high-cost anticancer drugs. Furthermore, quality and risk of bias of the included studies was not performed. Thus, methodological issues and inherent risk of bias of the included studies such as reporting bias might be presented. Finally, while this review could describe strategies in different countries, the impacts of these strategies on access to anticancer drugs could not be fully evaluated, especially in light of the large differences in the health systems of the identified countries. For example, the need for supplementary funding strategies specific to anticancer drugs depends on the basic reimbursement framework of a country, ie, if every drug is fully reimbursed, there is no need for other measures to facilitate access. A future study with a narrower scope could provide more information about the necessities, impacts, and effectiveness of the strategies described in specific country contexts.

\section{Conclusion}

Many countries have implemented a combination of strategies reflecting national health system objectives and affordability in order to increase access to high-cost anticancer drugs for their populations. MICs and LICs tend to facilitate patient access by listing anticancer medicines on the national list of essential medicines and insurance reimbursement lists, supplemented by pharmaceutical industry-initiated PAPs. In contrast, many HICs have developed targeted funding strategies for high-cost anticancer drugs that seek to address clinical uncertainties and the relatively poor $\mathrm{CE}$ of these drugs.

\section{Ethical issues}

Not applicable.

\section{Competing interests}

Authors declare that they have no competing interests.

\section{Authors' contributions}

Conception and design: CP, ST, RS, DRD, and PA. Acquisition of data: CP, ST, and PA. Analysis and interpretation of data: CP, ST, RS, DRD, and PA. Drafting of the manuscript: CP, ST, RS, DRD, and PA. Critical revision of the manuscript for important intellectual content: PA. Statistical analysis: CP. Obtaining funding: CP and PA. Administrative, technical, or material support: $\mathrm{CP}$ and PA. Supervision: PA.

\section{Data sharing}

The data that support the findings of this study are available from the corresponding author, PA, upon reasonable request.

\section{Funding}

This work was supported by the 90th Anniversary of Chulalongkorn University Scholarship Batch\#41 (4/2018) Grant number 31. The funder had no role in any part of the work including design and conduct of the study, data collection, data management, data analysis and interpretation, preparation, review and approval of the manuscript.

\section{Authors' affiliations}

${ }^{1}$ Department of Social and Administrative Pharmacy, Faculty of Pharmaceutical Sciences, Chulalongkorn University, Bangkok, Thailand. ${ }^{2}$ Department of Population Medicine, Harvard Medical School and Harvard Pilgrim Health Care Institute, Landmark Center, Boston, MA, USA.

\section{Supplementary files}

Supplementary file 1. PRISMA Checklist.

Supplementary file 2. Full Search Strategy With Results.

Supplementary file 3. Strategies to Facilitate Access to High-Cost Anticancer Drugs by Country.

\section{References}

1. Bray F, Ferlay J, Soerjomataram I, Siegel RL, Torre LA, Jemal A. Global cancer statistics 2018: GLOBOCAN estimates of incidence and mortality worldwide for 36 cancers in 185 countries. CA Cancer J Clin. 2018; 68(6):394-424. doi:10.3322/caac.21492

2. Ruiz R, Strasser-Weippl K, Touya D, et al. Improving access to highcost cancer drugs in Latin America: much to be done. Cancer. 2017 123(8):1313-1323. doi:10.1002/cncr.30549

3. Meropol NJ, Schrag D, Smith TJ, et al. American Society of Clinical Oncology guidance statement: the cost of cancer care. J Clin Oncol. 2009;27(23):3868-3874. doi:10.1200/jco.2009.23.1183

4. Leighl NB, Nirmalakumar S, Ezeife DA, Gyawali B. An arm and a leg: the rising cost of cancer drugs and impact on access. Am Soc Clin Oncol Educ Book. 2021;41:1-12. doi:10.1200/edbk_100028

5. de Lima Lopes G Jr, de Souza JA, Barrios C. Access to cancer medications in low- and middle-income countries. Nat Rev Clin Oncol. 2013;10(6):314322. doi:10.1038/nrclinonc.2013.55

6. Patikorn C, Taychakhoonavudh S, Thathong T, Anantachoti P. Patient access to anticancer medicines under public health insurance schemes in Thailand: a mixed methods study. Thai J Pharm Sci. 2019;43(3):168-178.

7. Ghinea N, Little M, Lipworth W. Access to high cost cancer medicines through the lens of an Australian senate inquiry-defining the "goods" at stake. J Bioeth Inq. 2017;14(3):401-410. doi:10.1007/s11673-017-9800-2

8. Faden RR, Chalkidou K, Appleby J, Waters HR, Leider JP. Expensive cancer drugs: a comparison between the United States and the United Kingdom. Milbank Q. 2009;87(4):789-819. doi:10.1111/j.14680009.2009.00579.x

9. Sruamsiri R, Ross-Degnan D, Lu CY, Chaiyakunapruk N, Wagner AK. Policies and programs to facilitate access to targeted cancer therapies in Thailand. PLoS One. 2015;10(3):e0119945. doi:10.1371/journal. pone.0119945

10. Pauwels K, Huys I, Casteels M, De Nys K, Simoens S. Market access of cancer drugs in European countries: improving resource allocation. Target Oncol. 2014;9(2):95-110. doi:10.1007/s11523-013-0301-x

11. Page MJ, McKenzie JE, Bossuyt PM, et al. The PRISMA 2020 statement: an updated guideline for reporting systematic reviews. BMJ. 2021; 372:n71. doi:10.1136/bmj.n71

12. World Bank. World Bank Country and Lending Groups. 2021; https:// datahelpdesk.worldbank.org/knowledgebase/articles/906519-worldbank-country-and-lending-groups. Accessed May 27, 2021.

13. Cherny N, Sullivan R, Torode J, Saar M, Eniu A. ESMO European Consortium Study on the availability, out-of-pocket costs and accessibility of antineoplastic medicines in Europe. Ann Oncol. 2016;27(8):1423-1443. doi:10.1093/annonc/mdw213

14. Cherny NI, Sullivan R, Torode J, Saar M, Eniu A. ESMO International Consortium Study on the availability, out-of-pocket costs and accessibility of antineoplastic medicines in countries outside of Europe. Ann Oncol. 2017;28(11):2633-2647. doi:10.1093/annonc/mdx521

15. Cuomo RE, Mackey TK. The availability of essential cancer medication: an analysis of national formularies. J Cancer Policy. 2017;12:49-54. doi:10.1016/j.jcpo.2017.03.010

16. Shafie AA, Chandriah $H$. Access to cancer drugs: are we meeting the needs of Malaysian? J Cancer Policy. 2017;13:30-32. doi:10.1016/j. jcpo.2017.07.003

17. Milliken D, Venkatesh J, Yu R, Su Z, Thompson M, Eurich D. Comparison of drug coverage in Canada before and after the establishment of the pan-Canadian Pharmaceutical Alliance. BMJ Open. 2015;5(9):e008100. doi:10.1136/bmjopen-2015-008100

18. Goss PE, Strasser-Weippl K, Lee-Bychkovsky BL, et al. Challenges to effective cancer control in China, India, and Russia. Lancet Oncol. 2014; 15(5):489-538. doi:10.1016/s1470-2045(14)70029-4

19. Menon D, Stafinski T, Stuart G. Access to drugs for cancer: does where you live matter? Can J Public Health. 2005;96(6):454-458. doi:10.1007/ bf03405189

20. World Health Organization (WHO). WHO Model List of Essential Medicines, 20th list (March 2017, Amended August 2017). WHO; 2017. 
21. Neumann PJ, Bliss SK, Chambers JD. Therapies for advanced cancers pose a special challenge for health technology assessment organizations in many countries. Health Aff(Millwood). 2012;31(4):700-708. doi:10.1377/ hlthaff.2011.1309

22. Karikios DJ, Chim L, Martin A, et al. Is it all about price? why requests for government subsidy of anticancer drugs were rejected in Australia. Intern Med J. 2017;47(4):400-407. doi:10.1111/imj.13350

23. Kim ES, Kim JA, Lee EK. National reimbursement listing determinants of new cancer drugs: a retrospective analysis of 58 cancer treatment appraisals in 2007-2016 in South Korea. Expert Rev Pharmacoecon Outcomes Res. 2017;17(4):401-409. doi:10.1080/14737167.2017.1276 828

24. Ferrario A. Time to entry for new cancer medicines: from European Union-wide marketing authorization to patient Access in Belgium, Estonia, Scotland, and Sweden. Value Health. 2018;21(7):809-821. doi:10.1016/j. jval.2018.01.003

25. McGuire A, Drummond M, Martin M, Justo N. End of life or end of the road? are rising cancer costs sustainable? is it time to consider alternative incentive and funding schemes? Expert Rev Pharmacoecon Outcomes Res. 2015;15(4):599-605. doi:10.1586/14737167.2015.1039518

26. Russo P, Mennini FS, Siviero PD, Rasi G. Time to market and patient access to new oncology products in Italy: a multistep pathway from European context to regional health care providers. Ann Oncol. 2010; 21(10):2081-2087. doi:10.1093/annonc/mdq097

27. Pauwels K, Huys I, Vogler S, Casteels M, Simoens S. Managed entry agreements for oncology drugs: lessons from the european experience to inform the future. Front Pharmacol. 2017;8:171. doi:10.3389/ fphar.2017.00171

28. Cohen J, Malins A, Shahpurwala Z. Compared to US practice, evidencebased reviews in Europe appear to lead to lower prices for some drugs. Health Aff (Millwood). 2013;32(4):762-770. doi:10.1377/hlthaff.2012.0707

29. Mihajlović J, Dolk C, Tolley K, Simoens S, Postma MJ. Reimbursement of targeted cancer therapies within 3 different European health care systems. Clin Ther. 2015;37(2):474-480. doi:10.1016/j.clinthera.2014.12.005

30. Ferrario A, Arāja D, Bochenek T, et al. The implementation of managed entry agreements in Central and Eastern Europe: Findings and Implications. Pharmacoeconomics. 2017;35(12):1271-1285. doi:10.1007/ s40273-017-0559-4

31. Grover P, Babar ZU, Oehmen R, Vitry A. Medicines access programs to cancer medicines in Australia and New Zealand: an exploratory study. Health Policy. 2018;122(3):243-249. doi:10.1016/j.healthpol.2017.12.004

32. Blommestein HM, Franken MG, Verelst SG, van Agthoven M, Huijgens PC, Uyl-de Groot CA. Access to expensive cancer drugs in Dutch daily practice: should we be concerned? Neth J Med. 2014;72(4):235-241.

33. Pace J, Ghinea N, Kerridge I, Lipworth W. Demands for access to new therapies: are there alternatives to accelerated access? BMJ. 2017; 359:j4494. doi:10.1136/bmj.j4494

34. Zhang Y, Hueser HC, Hernandez I. Comparing the approval and coverage decisions of new oncology drugs in the United States and other selected countries. J Manag Care Spec Pharm. 2017;23(2):247-254. doi:10.18553/ jmcp.2017.23.2.247

35. Morrell L, Wordsworth S, Fu H, Rees S, Barker R. Cancer drug funding decisions in Scotland: impact of new end-of-life, orphan and ultra-orphan processes. BMC Health Serv Res. 2017;17(1):613. doi:10.1186/s12913017-2561-0

36. Löblová O, Csanádi M, Ozierański $\mathrm{P}$, Kaló Z, King L, McKee M. Patterns of alternative access: unpacking the Slovak extraordinary drug reimbursement regime 2012-2016. Health Policy. 2019;123(8):713-720. doi:10.1016/j.healthpol.2019.05.021

37. den Exter A. Fighting excessive pharmaceutical prices: evaluating the options. Eur J Health Law. 2020;28(1):68-80. doi:10.1163/15718093bja10004

38. Haitsma G, Patel H, Gurumurthy P, Postma MJ. Access to anti-cancer drugs in India: is there a need to revise reimbursement policies? Expert Rev Pharmacoecon Outcomes Res. 2018;18(3):289-296. doi:10.1080/14 737167.2018.1444479

39. Dusetzina SB, Keating NL. Mind the gap: why closing the doughnut hole is insufficient for increasing Medicare beneficiary access to oral chemotherapy. J Clin Oncol. 2016;34(4):375-380. doi:10.1200/ jco.2015.63.7736

40. Lassarat S, Jootar S. Ongoing challenges of a global international patient assistance program. Ann Oncol. 2006;17 Suppl 8:viii43-viii46. doi:10.1093/annonc/mdl987

41. Umeh CA, Garcia-Gonzalez P, Tremblay D, Laing R. The survival of patients enrolled in a global direct-to-patient cancer medicine donation program: The Glivec International Patient Assistance Program (GIPAP). EClinicalMedicine. 2020;19:100257. doi:10.1016/j.eclinm.2020.100257

42. Lee AW, Sze H, Lam KO, Chen X. A tale of two cities in China: Hong Kong and Shenzhen. Int J Radiat Oncol Biol Phys. 2014;89(4):704-708. doi:10.1016/j.ijrobp.2013.12.053

43. Egerton NJ. In-office dispensing of oral oncolytics: a continuity of care and cost mitigation model for cancer patients. Am J Manag Care. 2016;22(4 Suppl):S99-S103.

44. Mitchell A, Muluneh B, Patel R, Basch E. Pharmaceutical assistance programs for cancer patients in the era of orally administered chemotherapeutics. J Oncol Pharm Pract. 2018;24(6):424-432. doi:10.1177/1078155217719585

45. Lakdawalla DN, Doshi JA, Garrison LP Jr, Phelps CE, Basu A, Danzon PM. Defining elements of value in health care-a health economics approach: an ISPOR Special Task Force report [3]. Value Health. 2018;21(2):131139. doi:10.1016/j.jval.2017.12.007 